Ann. Génét. Sél. anim., I970, 2 (3), 3II-324.

\title{
L'ÉPREUVE DE LA DESCENDANCE CHEZ LE PORC LARGE WHITE FRANÇAIS DE 1953 A 1966
}

\author{
I. - ANALYSE DE LA VARIATION
}

L. OLLIVIER

avec la collaboration technique de $\mathrm{N}$. Boutler, D. Brault, M. Renault et du Service de calcul de la Station centrale de Génétique animale

Station de Génétique quantitative et appliquée, Centre national de Recherches zootechniques, 78-Jouy-en-Josas

Institut national de la Recherche agronomique

\section{RÉSUMÉ}

A partir d'un échantillon de 2657 femelles et de 1515 mâles castrés, les paramètres génétiques, héritabilités et corrélations génétiques, de la race porcine Large White française ont été estimés pour la consommation d'aliment et la durée d'engraissement de 25 à 100 kilogrammes de poids vif, le poids de différents morceaux et des mesures de longueur et d'épaisseur du lard sur la carcasse au poids vif d'abattage de 100 kilogrammes. Les estimations obtenues sont très voisines de celles de la même race exploitée en Grande-Bretagne.

Une analyse en composantes principales des matrices de corrélations phénotypiques et génétiques a permis de dégager une première composante "adiposité de la carcasse "et une deuxième composante "vitesse de croissance et consommation d'aliment ". Les résultats de l'analyse phénotypique et génétique sont similaires et peu différentes entre mâles et femelles.

\section{INTRODUCTION}

Le Pr LEROy avait proposé, dès I928, de créer en France des stations pour la mise à l'épreuve des verrats sur leur descendance, à l'exemple du Danemark. Après une période expérimentale, de 1947 à 1952 à Bois-Corbon, une station plus importante fut créée par l'I.N.R.A. à Jouy-en-Josas et c'est en I953 que l'épreuve de la descendance fut réellement mise à la disposition des sélectionneurs français. Cette technique de sélection a été souvent discutée, tant du point de vue théorique que de celui de son utilisation pratique (voir, en particulier, DICKER- 
son et Hazei, r944; Smith, r965; Czajewska et Olitivier, r969). Il semble que l'intérêt de ces porcheries à grande capacité, où l'on s'efforce de normaliser les conditions de milieu, est double. D'abord, elles nous fournissent une information sur l'état des populations porcines à un instant donné, sous forme de divers paramètres phénotypiques et génétiques. Ces paramètres ont une grande importance pratique car ils sont à la base du calcul des indices de sélection. D'autre part, les résultats recueillis dans ces conditions reflètent l'évolution au cours des années des caractères que l'on veut améliorer. Ce sont les deux aspects qui seront abordés dans ce mémoire, pour le cas de la race porcine Large White exploitée en France.

\section{MATÉRIEL ANIMAL - MESURES EFFECTUÉES}

Les données utilisées dans cette étude concernent 4172 porcs de race Large. White (2 657 femelles et 1515 mâles) engraissés, de 1953 à 1966 inclus, dans les deux porcheries de mise à l'épreuve des verrats sur leur descendance, situées au C.N.R.Z., l'une à Jouy-en-Josas, l'autre sur le domaine de la Minière. L'éleveur désirant mettre un verrat à l'épreuve devait soumettre aux contrôles au moins 3 lots d'animaux, comprenant chacun 3 porcs choisis par lui dans une portée, et pesant à leur arrivée entre 20 et 25 kilogrammes de poids vif. Jusqu'à 1956 , chaque lot comprenait 1 femelle et 2 mâles castrés. A partir de 1956 le rapport a été inversé.

Les porcs étaient placés en loges individuelles à partir d'un poids vif voisin de 25 kilogrammes, et alimentés en régime semi-ad-libitum, c'est-à-dire qu'ils recevaient à chaque repas une quantité d'aliment correspondant à ce qu'ils pouvaient ingérer en 20 à 30 minutes, le nombre de repas étant de trois par jour. L'aliment était distribué sous forme de farine, légèrement humidifiée au début du repas, avec un apport supplémentaire d'eau vers la fin du repas. La composition énergétique et azotée a peu varié de 1953 à 1966. Les mélanges alimentaires avaient les compositions suivantes :

$$
\begin{array}{cc}
\text { Aliment " Croissance " Aliment " Finition " } \\
\text { (de } 25 \text { à } 60 \mathrm{~kg}) & \text { (de 6o à Ioo kg) }
\end{array}
$$

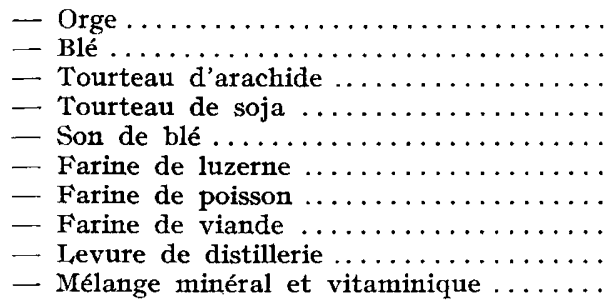

$\begin{array}{rr}38 & 4 \mathbf{I} \\ 38 & 42 \\ 4 & 3 \\ 4 & 3 \\ 5 & - \\ -2 & 5 \\ 2 & -2 \\ 4 & 2 \\ 3 & 2\end{array}$

Les porcs étaient pesés, le matin à jeûn, tous les quatorze jours et les consommations individuelles journalières étaient enregistrées. L'abattage avait lieu à un poids vif voisin de $100 \mathrm{kilo-}$ grammes. De 1953 à 1962, l'abattage se faisait au C.N.R.Z. Depuis 1963, l'abattage a lieu aux Établissements G.E.O., au Kremlin-Bicêtre. Le lendemain de l'abattage la carcasse était pesée d'une part (poids net) et la tête (avec les joues) d'autre part. La moitié droite de chaque carcasse était ensuite soumise à une découpe " parisienne "normalisée dont voici le principe (voir fig. 1). La demi-carcasse est posée à plat sur une table, face externe contre la table. Les coupes sont effectuées perpendiculairement à la table. La panne (gras périrénal) est d'abord séparée de la cavité abdominale; avant sa pesée on enlève la hampe et le rein. Le pied arrière est séparé du membre postérieur par une coupe ABG effectuée au niveau de l'articulation tibio-tarsienne. Le pied avant est séparé du membre antérieur par une coupe $A^{\prime} B^{\prime}$ effectuée à la scie au niveau des carpiens. Le jambon est isolé de la carcasse par section des muscles et des os au niveau des deux lignes de coupe DE et EF. La direction de la ligne DE est obtenue en traçant la ligne $\mathrm{DD}^{\prime}$ qui passe entre la dernière vertèbre lombaire et la première vertèbre sacrée et qui est perpendiculaire à la ligne du dessous. La ligne EF est tracée parallèlement à la direction générale du sacrum et passe par un point $E^{\prime}$ équidistant du bord antérieur de la symphyse pubienne et du bord postérieur de la dernière vertèbre lombaire. La longe est séparée de la poitrine par une coupe GHE. Cette ligne longe la "veine grasse ", le défaut de l'épaule pour atteindre le point E. La bardière est séparée de la longe au couteau de façon à laisser le moins possible de gras sur la longe. L'ensemble poitrine-hachage-jambonneau était, de 1953 à 1962, partagé en poitrine-hachage d'une part 
et jambonneau d'autre part. Depuis 1963 , la poitrine est séparée de l'ensemble hachage-jambonneau par une coupe $\mathrm{HH}^{\prime}$ effectuée au couteau entre la $5^{\mathrm{e}}$ et la $6^{\mathrm{e}}$ côte. Dans cette étude nous considérons la somme de ces deux morceaux. Les mesures suivantes étaient, d'autre part, effectuées sur la demi-carcasse gauche suspendue : longueur mesurée entre l'atlas et le bord antérieur de la symphyse pubienne, épaisseurs de lard au niveau de la dernière vertèbre lombaire (rein), de la dernière vertèbre dorsale (dos) et de la dernière vertèbre cervicale (cou).

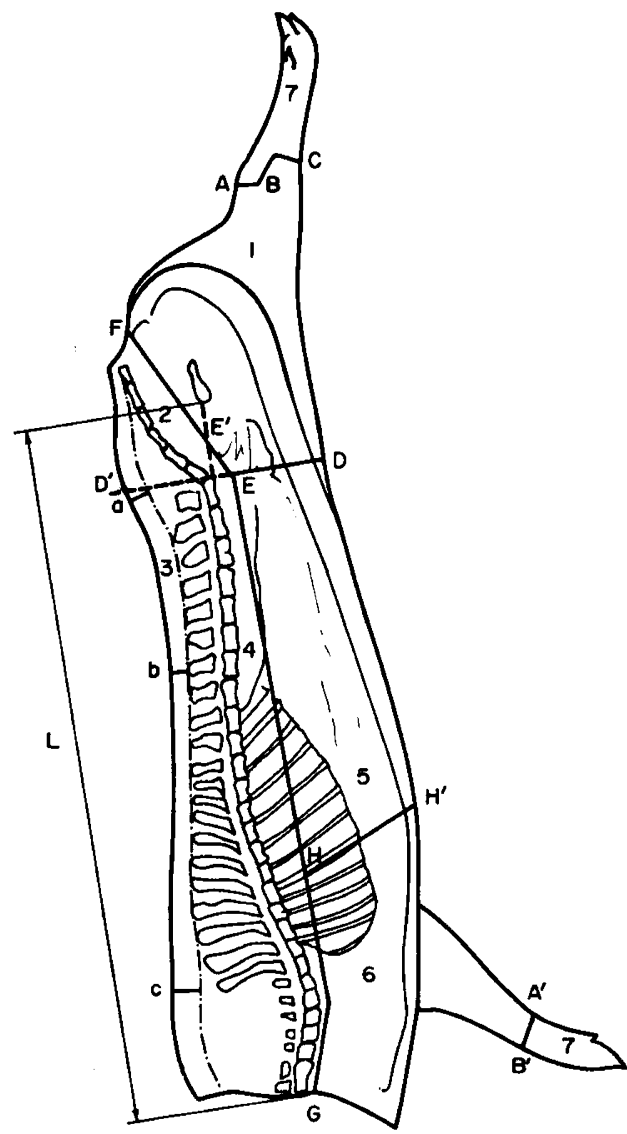

Légende :
I. Jambon
a. Rein
2. Longe
b. Dos
3. Bardière
c. Cou
4. Panne
L. Longueur
6. Hachage et jambonneau
7. Pieds

FIG. 1. - Découpe parisienne normalisée et mesures prises sur la carcasse (Dessin de O. Schmit)

En résumé, les mesures suivantes ont été considérées dans cette étude :

1. Poids net

2. Poids de la tête

3. Poids du jambon

4. Poids de la longe

5. Poids de l'ensemble poitrine-hachage-jambonneau

6. Poids des pieds

7. Poids de la bardière

8. Poids de la panne

9. Longueur

10. Épaisseur de lard au rein

11. Épaisseur de lard au dos

12. Épaisseur de lard au cou

13. Consommation d'aliment pendant la période de contrôle

14. Durée de la période de contrôle. 


\section{MÉTHODE D'ANALYSE}

Les variables énumérées ci-dessus ont été d'abord corrigées pour les variations de poids initial, d'âge initial et de poids vif d'abattage, soit par régression simple

TABLEAU I

Moyennes et écarts-types de l'échantillon étudié

\begin{tabular}{|c|c|c|c|c|}
\hline \multirow{3}{*}{ Effectif } & \multicolumn{2}{|c|}{ Femelles } & \multicolumn{2}{|c|}{ Mâles } \\
\hline & \multicolumn{2}{|c|}{2657} & \multicolumn{2}{|c|}{ I 5 I 5} \\
\hline & Moyenne & Écart-type & Moyenne & Écart-type \\
\hline 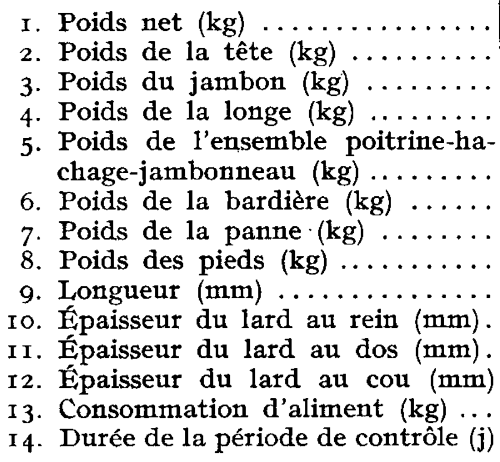 & $\begin{array}{l}71, I \\
5,34 \\
8,06 \\
10,72 \\
9,16 \\
5,16 \\
0,75 \\
1,05 \\
988,1 \\
26,3 \\
23,4 \\
43,4 \\
259,6 \\
120,0\end{array}$ & $\begin{array}{c}2,12 \\
0,39 \\
0,42 \\
0,60 \\
0,58 \\
0,70 \\
0,18 \\
0,09 \\
2,80 \\
5,00 \\
4,25 \\
5,40 \\
24,8 \\
14,5\end{array}$ & $\begin{array}{r}71,4 \\
5,23 \\
7,82 \\
\text { I0,24 } \\
\\
9, \text { I } 5 \\
5,99 \\
0,84 \\
1,05 \\
975,4 \\
30,6 \\
26,8 \\
46,0 \\
267,0 \\
\text { I } 7,1\end{array}$ & $\begin{array}{l}2,18 \\
0,39 \\
0,43 \\
0,59 \\
0,62 \\
0,77 \\
0,20 \\
0,14 \\
2,79 \\
5,32 \\
4,46 \\
5,59 \\
26,1 \\
14,0\end{array}$ \\
\hline
\end{tabular}

TABLEAU 2

Coefficients de régression des mesures de carcasse en poids vif $(\mathrm{kg})$

\begin{tabular}{|c|c|c|}
\hline & Femelles & Mâles \\
\hline 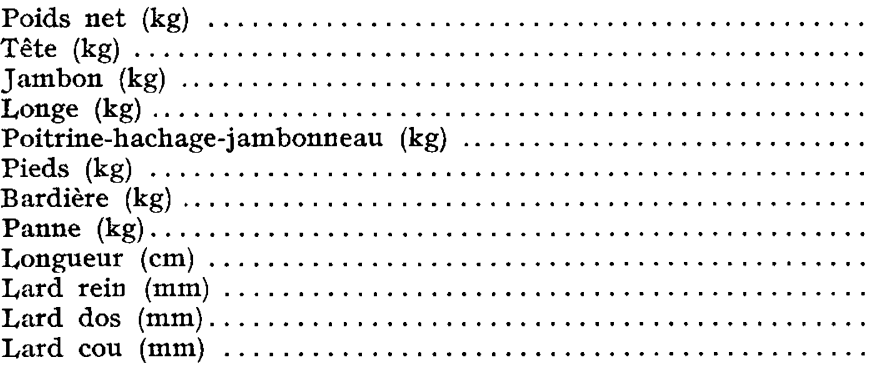 & $\begin{array}{l}0,626\left(^{2}\right) \\
0,024 \\
0,064 \\
0,081 \\
0,085\left(^{1}\right) \\
0,006 \\
0,064 \\
0,006 \\
0,28 \\
0,19 \\
0,26 \\
0,28\end{array}$ & $\begin{array}{l}0,604{ }^{(1)} \\
0,029 \\
0,073 \\
0,076 \\
0,076 \\
0,006 \\
0,064 \\
0,006 \\
0,36 \\
0,16 \\
0,25 \\
0,23\end{array}$ \\
\hline
\end{tabular}

(1) Hypothèse de linéarité rejetée au seuil de $5 \mathrm{p}$. Ioo.

${ }^{2}$ ) Hypothèse de linéarité rejetée au seuil de $\mathrm{I}$ p. Ioo. 
des mesures de carcasse en poids vif d'abattage (tabl. 2), soit par régression multiple de la consommation et de la durée d'engraissement en poids initial, gain de poids et âge initial (tabl. 3). Les variables I à I2 ont ainsi été ramenées à un

TABLEAU 3

Coefficient de régression partielle de la consommation alimentaire (en $\mathrm{kg}$ ) et de la durée d'engraissement (en j) en :

$$
\begin{aligned}
& x_{1}-\text { poids initial (kg) } \\
& x_{2}-\text { gain de poids (kg) } \\
& x_{3}-\text { age initial (j) }
\end{aligned}
$$

\begin{tabular}{|c|c|c|c|c|}
\hline & & $x_{1}$ & $x_{2}$ & $x_{3}$ \\
\hline \multirow{2}{*}{ Consommation } & Femelles & 0,19 & 1,85 & $-0,05$ \\
\hline & Mâles ... & 0,43 & 2,78 & $-0, \infty$ \\
\hline \multirow{2}{*}{$\begin{array}{c}\text { Durée } \\
\text { d'engraissement }\end{array}$} & Femelles & $-0,87$ & 0,23 & $-0,15$ \\
\hline & Mâles ... & $-0,12$ & $\mathrm{I}, \mathrm{O3}$ & $-0,09$ \\
\hline
\end{tabular}

poids vif d'abattage de Ioo kilogrammes, et les variables I3 et I4 à un poids initial de 25 kilogrammes, un âge initial de 85 jours et un poids d'abattage de roo kilogrammes. Ces corrections ont été faites séparément dans les deux sexes.

L'analyse de la variation a été effectuée séparément chez les mâles et les femelles après classification des données selon une hiérarchie année-station, père, mère. La méthode d'analyse des variances et covariances dans le cas d'une telle classification a été décrite par KEMPTHORNE (I957). Nous avons utilisé un programme de calcul sur ordinateur IBM I620 établi par la Station centrale de Génétique animale (VISSAc et coll., I962). Il n'a pas été possible de prendre en considération l'effet de l'élevage d'origine des animaux, qui se trouve ainsi confondu avec l'effet " père ". La variance génétique additive de chaque caractère a été estimée en multipliant par 4 la composante paternelle de la variance, ce qui suppose l'absence de parenté entre les différentes truies accouplées à un même verrat et l'absence de consanguinité chez les descendants contrôlés. Les héritabilités ont été calculées en divisant les variances génétiques additives par les variances entre animaux contrôlés dans la même année et la même porcherie (variance phénotypique). Les corrélations phénotypiques ont été estimées également entre animaux contrôlés dans la même année et la même station. Les corrélations génétiques ont été estimées à partir des composantes paternelles des variances et des covariances. Pour évaluer la précision des estimations obtenues, la variance de l'estimateur d'héritabilité a été calculée par la méthode-de Woolf décrite par 
Fatconer ( $\mathrm{r}_{963}$ ), et celle de l'estimateur de corrélation génétique par la méthode de FALCONER ( 1960 ).

Les matrices des corrélations phénotypiques et génétiques ont été analysées par la méthode des composantes principales (HotELLIING, I933), déjà utilisée chez le Porc par Smith et coll. (I962), chez les bovins par TAYLor et Rolins (I963) et chez le poulet par Rouvier et RICARD (I965).

\section{RÉSULTATS}

Les composantes des variances et les héritabilités figurent au tableau 4 . La composante paternelle représente le quart de la variance génétique additive. La composante maternelle inclut, en plus, la variance due aux effets de dominance

TABLEAU 4

Décomposition de la variance

\begin{tabular}{|c|c|c|c|c|c|c|c|}
\hline \multirow[b]{2}{*}{ Variable } & \multirow[b]{2}{*}{ Sexe } & \multicolumn{4}{|c|}{ Composantes de variance } & \multirow{2}{*}{$\begin{array}{c}\text { Hérita- } \\
\text { bilité } \\
h^{2}-{ }^{4} C / \\
(A+ \\
B+C)\end{array}$} & \multirow{2}{*}{$\begin{array}{l}\text { Écart- } \\
\text { type } \\
\text { de } h^{2}\end{array}$} \\
\hline & & $\begin{array}{l}\text { Indi- } \\
\text { vidu } \\
(A)\end{array}$ & $\begin{array}{l}\text { Mère } \\
(B)\end{array}$ & $\begin{array}{l}\text { Père } \\
(C)\end{array}$ & $\begin{array}{l}\text { Année- } \\
\text { station } \\
(D)\end{array}$ & & \\
\hline \multirow[t]{2}{*}{ I. Poids net $(\mathrm{kg})$} & $\mathrm{F}$ & I,696 & 0,327 & 0,314 & 0,688 & 0,54 & 0,10 \\
\hline & M & $\mathrm{I}, 975$ & 0,224 & 0,278 & 0,762 & 0,45 & 0,13 \\
\hline \multirow[t]{2}{*}{ 2. Poids de la tête $(\mathrm{kg}) \ldots \ldots$} & $\mathrm{F}$ & $0,07 I_{4}$ & 0,0236 & 0,0140 & 0,0429 & $0,5^{\mathrm{I}}$ & $0, \mathrm{I} \mathrm{I}$ \\
\hline & $\mathbf{M}$ & 0,0678 & 0,0184 & O,OIOI & 0,0476 & 0,42 & 0,13 \\
\hline \multirow{2}{*}{ 3. Poids du jambon $(\mathrm{kg}) \ldots \ldots$} & $\mathrm{F}$ & 0,1047 & 0,0190 & 0,0137 & 0,0209 & $0,4^{\circ}$ & 0,10 \\
\hline & $\mathbf{M}$ & 0,0756 & 0,0556 & 0,0107 & 0,0231 & 0,30 & 0,12 \\
\hline \multirow{2}{*}{ 4. Poids de la longe $(\mathrm{kg}) \ldots \ldots$} & $\mathbf{F}$ & 0,2126 & 0,0418 & 0,0435 & 0,0432 & $0,5^{8}$ & 0, I I \\
\hline & M & 0,2624 & négative & 0,0543 & 0,0280 & 0,73 & 0,13 \\
\hline \multirow{4}{*}{$\begin{array}{l}\text { 5. Poids de l'ensemble poitrine- } \\
\text { hachage-jambonneau }(\mathrm{kg}) \ldots \\
\text { 6. Poids des pieds }(\mathrm{kg}) \ldots \ldots \ldots\end{array}$} & $\mathbf{F}$ & $0,155^{8}$ & 0,0237 & 0,0306 & 0,0674 & $0,5^{8}$ & 0,10 \\
\hline & M & 0,1882 & 0,0075 & 0,0122 & 0,0867 & 0,23 & 0,12 \\
\hline & $\mathbf{F}$ & 0,0049 & 0,0010 & 0,0012 & 0,0003 & 0,70 & 0,10 \\
\hline & $\mathbf{M}$ & 0,0056 & 0,0009 & 0,001 I & 0,0006 & $0,5^{8}$ & 0,13 \\
\hline \multirow[t]{2}{*}{ 7. Poids de la bardière $(\mathrm{kg})$.. } & $\mathbf{F}$ & 0,2645 & 0,1160 & 0,0768 & $0,022 \mathrm{I}$ & 0,67 & $0, \mathrm{II}$ \\
\hline & M & 0,3318 & 0,1008 & 0,1054 & 0,0346 & 0,78 & 0,13 \\
\hline \multirow{4}{*}{$\begin{array}{l}\text { 8. Poids de la panne }(\mathrm{kg}) \ldots \\
\text { 9. Longueur atlas-pubis }(\mathrm{mm}) \text {. }\end{array}$} & $\mathbf{F}$ & 0,0166 & $0,007 \mathrm{I}$ & 0,0068 & 0,0029 & 0,89 & $0, \mathbf{I}$ I \\
\hline & M & 0,0184 & 0,0084 & 0,0099 & 0,0043 & $\mathrm{I}, 08$ & 0,13 \\
\hline & $\mathbf{F}$ & 400 & I I I I & I24 & II 6 & 0,78 & $0, \mathrm{II}$ \\
\hline & M & 4I 4 & 89 & 100 & I I 3 & 0,66 & 0,13 \\
\hline \multirow{2}{*}{ I0. Épaisseur du lard au rein } & F & $\mathrm{x} 3,83$ & 6,93 & 3,10 & I,25 & 0,52 & 0,12 \\
\hline & M & I6,26 & 5,32 & 5,54 & 1,26 & 0,82 & 0,13 \\
\hline \multirow{6}{*}{ 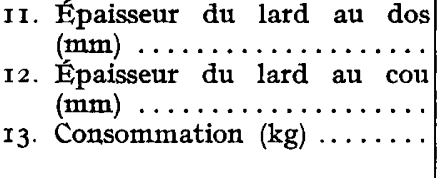 } & $\mathrm{F}$ & II, $4 \mathrm{I}$ & 2,80 & 2,76 & 0,83 & 0,65 & 0,10 \\
\hline & M & I 2,25 & 2,86 & 4,09 & 0,45 & 0,85 & 0,13 \\
\hline & $F$ & I9,76 & 3,96 & 2,48 & $\mathrm{I}, 39$ & 0,38 & 0,10 \\
\hline & $\mathbf{M}$ & I3,I I & I 2, I I & 4,10 & I,66 & 0,56 & 0,13 \\
\hline & F & 215,5 & 68,2 & 32,5 & 245,0 & $0,4 \mathrm{I}$ & $0, \mathrm{II}$ \\
\hline & M & 138,5 & I 59,7 & 47,6 & 307,8 & 0,55 & 0,13 \\
\hline \multirow{2}{*}{ 14. Durée d'engraissement $(\mathrm{j})$.. } & $\mathbf{F}$ & $\mathrm{r} I 3,5$ & 34,7 & 23,7 & 44,0 & 0,55 & 0,13 \\
\hline & M & 63,6 & 60,7 & I9,3 & 56,8 & 0,54 & 0,13 \\
\hline
\end{tabular}




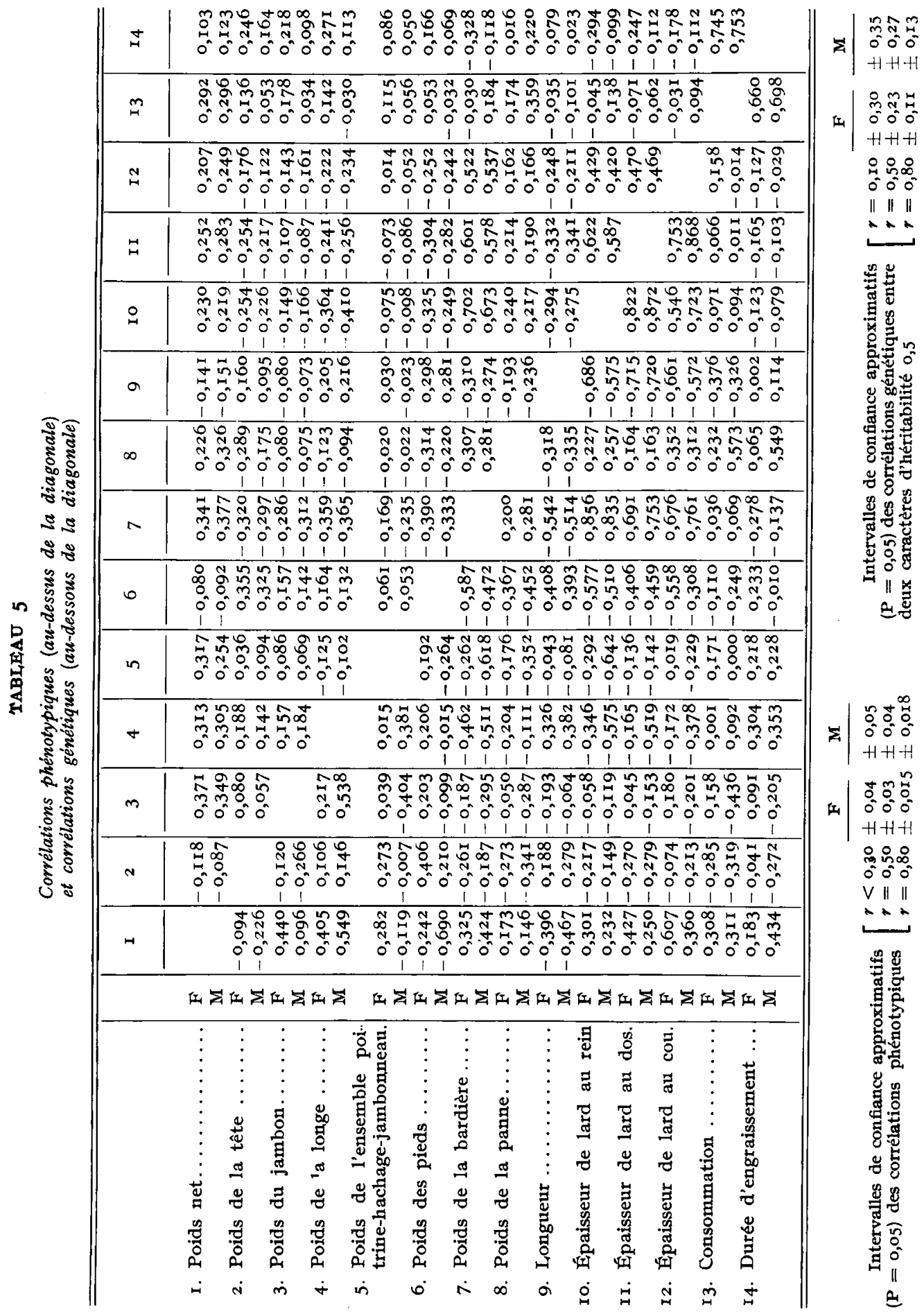


et de milieu commun aux individus d'une même portée. On devrait donc, généralement, obtenir une composante maternelle supérieure à la composante paternelle. Le tableau 4 montre que c'est toujours le cas pour les performances d'engraissement (variables $\mathrm{I}_{3}$ et $\mathrm{I}_{4}$ ) mais que pour les caractères de carcasse (variables I à I2) les différences sont moins importantes et, parfois même, la composante maternelle est inférieure à la composante paternelle. Cela suggère que les effets "portée " sont sensibles sur les critères de croissance (et peut-être aussi les effets de dominance) mais qu'ils sont très atténués sur lescritères mesurés à 1'abattage. Les estimations d'héritabilité vont de 0,23 à $\mathrm{I}, 08$ et sont en moyenne plus élevées pour les mesures de carcasse que pour les critères de croissance. Les différences entre sexes sont peu importantes. On note cependant des valeurs constamment supérieures de l'héritabilité chez les mâles pour les mesures d'adiposité (variable 7, 8 , IO, II, I2).

Les corrélations phénotypiques (tabl. 5) peuvent être considérées comme significativement différentes de o à partir de $\pm 0,04$ chez les femelles et $\pm 0,05$ chez les mâles. Les corrélations entre les performances d'engraissement considérées 2 à 2 d'une part et entre les performances de carcasse considérées 2 à 2 d'autre part sont relativement élevées et remarquablement voisines dans les deux sexes. Par contre, les corrélations entre ces deux ensembles de variables sont plus faibles et révèlent en outre de légères différences entre les sexes. Chez les femelles une croissance rapide s'accompagne de carcasses plus grasses, alors que l'indice de consommation est pratiquement indépendant de l'adiposité de la carcasse. Au contraire, chez les mâles, la vitesse de croissance est moins liée à l'adiposité et l'indice de consommation semblerait plus étroitement lié à ce caractère que chez les femelles.

Les corrélations génétiques (tabl. 5) sont estimées avec une précision moindre que les corrélations phénotypiques, si bien qu'aucune des différences observées entre les sexes n'est significative. Les corrélations génétiques sont, dans l'ensemble, du même ordre de grandeur que les corrélations phénotypiques.

L'analyse en composantes principales permet de dégager une série de fonctions linéaires des différents caractères. Les corrélations entre ces fonctions sont nulles et elles sont classées dans l'ordre décroissant de leur variance. La variable I (poids net) n'a pas été considérée car elle est la somme des variables 3 à 8 . Les matrices des corrélations phénotypiques et génétiques pour chaque sexe ont été analysées séparément. Seules les deux premières composantes, qui rendent compte à elles seules d'environ 50 p. roo de la variance totale, ont été retenues dans le tableau 6 , qui donne les corrélations de chaque mesure avec ces deux composantes. Les résultats sont similaires dans les quatre analyses réalisées, ce qui confirme l'absence de différences importantes entre sexes dans les matrices de corrélations et aussi l'analogie entre les corrélations génétiques et phénotypiques, signalée plus haut. La première composante correspond à un facteur d'adiposité de la carcasse. Elle est en corrélation plus étroite avec la bardière qu'avec la panne et, parmi les mesures d'épaisseur du lard dorsal, c'est la mesure au cou qui est la moins associée à ce facteur. La deuxième composante correspond à un facteur de croissance et d'efficacité alimentaire, facteur peu lié aux mesures de carcasse, dans leur ensemble. Remarquons cependant qu'il tend à être associé au poids de la 
TABLEAU 6

Coefficients de corrélation entre les $x_{3}$ mesures et les 2 premières composantes principales

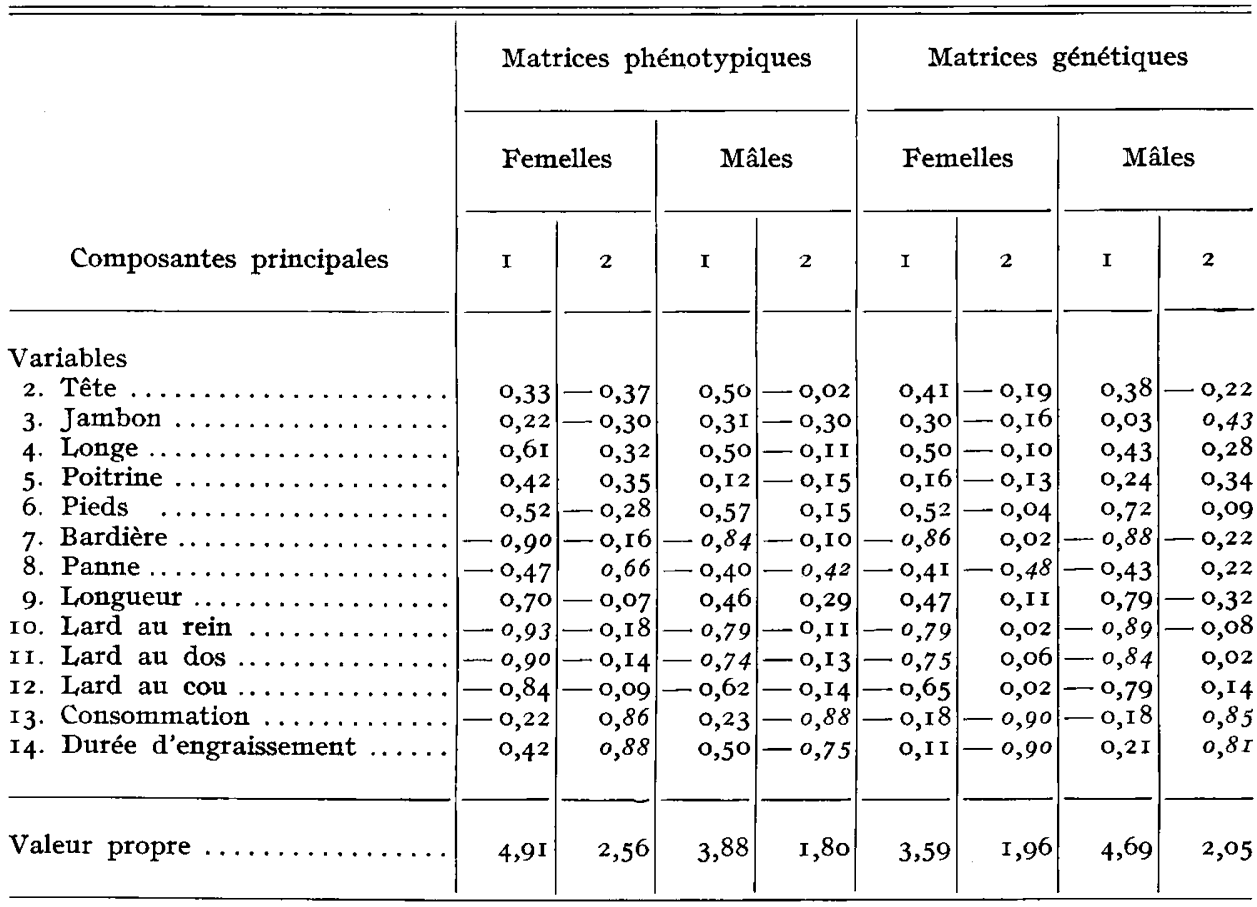

(Les 3 corrélations les plus élevées pour chaque composante sont en italique.)

panne et, pour les valeurs génétiques chez les mâles, au poids du jambon. La figure 2 donne une représentation graphique de la position des différentes mesures dans un système d'axes de coordonnées correspondant aux deux premières composantes. Chaque mesure est représentée par un point dont les coordonnées sont les corrélations entre cette mesure et les deux composantes considérées. Le segment de droite joignant 1'origine à ce point donne une idée pour chaque mesure du rôle qu'elle joue dans les deux composantes considérées. Ainsi les mesures d'adiposité (variables 7, IO, II, I2) apparaissent, dans les quatre cas, bien groupées et s'opposent au poids de longe (variable 4) et à la longueur (variable 9). De même la durée d'engraissement (variable I4) et la consommation (variable I3) jouent des rôles similaires dans la deuxième composante et apparaissent peu liés à la première. Les poids des différents morceaux, à l'exception de la longe et de la bardière, occupent des positions assez variables et ne se rattachent pas systématiquement à l'une ou à l'autre de ces deux composantes. Les composantes suivantes sont assez différentes dans les quatre analyses et il est difficile d'y discerner une que1conque tendance générale. 


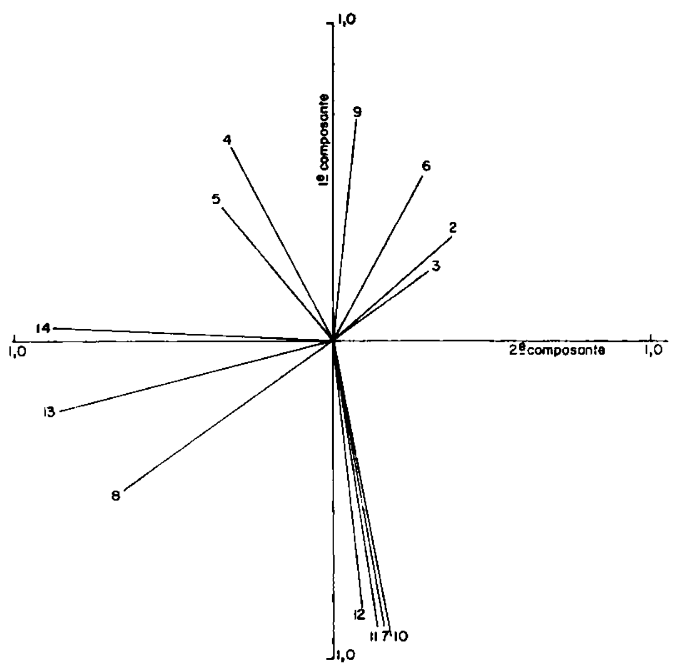

$2 a$

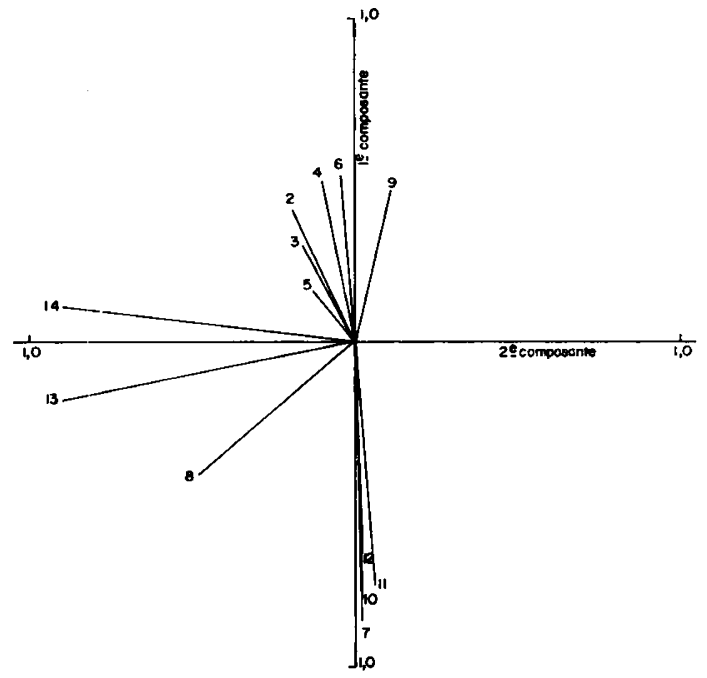

$2 c$

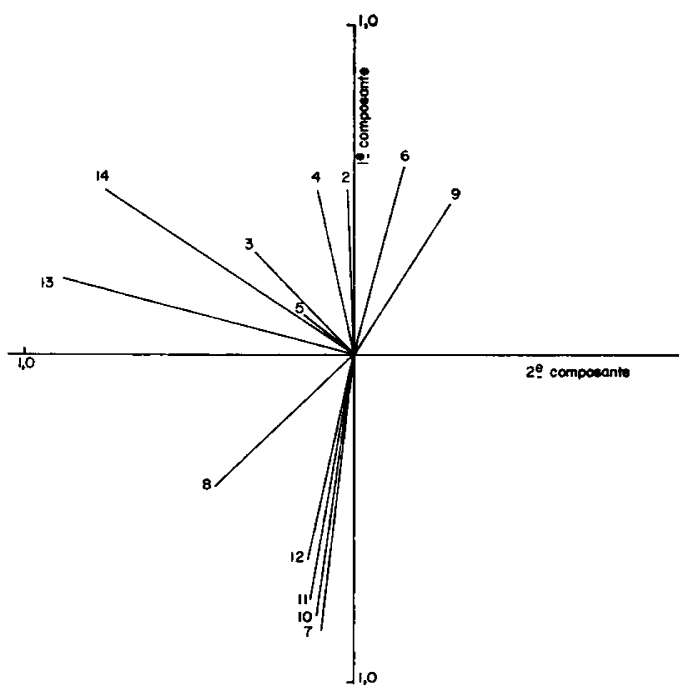

$2 b$

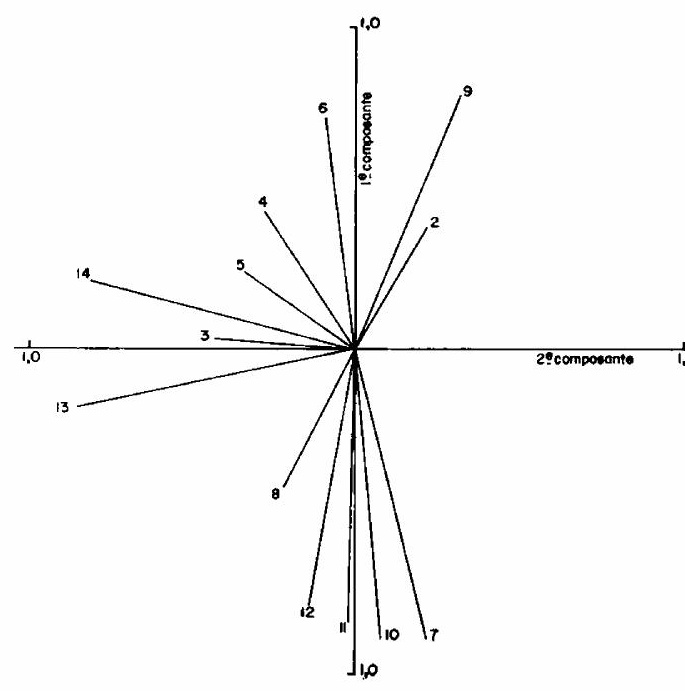

$2 d$

FIG. 2. - Diagramme des vecteurs des deux premieres composantes principales (Les chiffres correspondent aux numéros des variables du tableau 6)

2a. Corrélations phénotypiques (femelles)

2b. Corrélations phénotypiques (mâles)

2c. Corrélations génétiques (femelles)

$2 d$. Corrélations génétiques (mâles). 


\section{DISCUSSION ET CONCLUSIONS}

Les estimations de paramètres génétiques qui viennent d'être présentées reposent sur un certain nombre d'hypothèses qu'il est difficile de vérifier mais qui doivent être admises dans l'état actuel de nos connaissances et de nos possibilités de calcul. Nous avons d'abord négligé l'effet de l'élevage d'origine des animaux contrôlés. C'est une hypothèse que l'on peut admettre car une étude précédente (OLLIVIER, Ig69) a démontré que l'âge à 25 kilogrammes, qui reflète ces influences d'élevage, a finalement peu d'incidence sur l'ensemble des caractères considérés. D'autre part, l'effet de l'élevage a pu être estimé au Danemark par LAUPRECHT et WALTER (I960) et JoNSSON et KING (I962), et en Norvège par LANGHOLZ (I966). Ces auteurs s'accordent pour reconnaître la faible importance de cette cause de variation qui peut expliquer, au plus, 5 p. roo de la variance totale.

Il n'a pas été tenu compte non plus des fluctuations des conditions de milieu au cours de l'année, fluctuations occasionnées soit par la saison, soit par des événements aléatoires liés par exemple à l'état sanitaire. LANGHOLZ (I965) a utilisé la méthode des moyennes mobiles de station pour éliminer cette source de variation. Les valeurs d'héritabilité qu'il obtient ainsi sont, dans l'ensemble, inférieures à celles obtenues dans les analyses de variance classiques (LANGHOLZ, I966). Il est donc probable que les héritabilités du tableau 4 sont surestimées, du fait que la composante père inclut d'éventuels effets de l'élevage d'origine ainsi qu'une portion des fluctuations saisonnières.

La structure génétique de la population conditionne également la validité de nos estimations. La méthode que nous avons employée suppose la panmixie. Il est théoriquement possible de tenir compte de la structure génétique réelle, en utilisant l'information sur les diverses chaînes de parenté entre les individus considérés (GILI,OIS, I964 et HARRIS, I964). Mais la collecte de cette information est très laborieuse et le nombre des paramètres génétiques à estimer est considérablement accru. FLOCK (I967) a proposé une méthode simplifiée pour tenir compte d'une parenté possible entre les différentes truies accouplées au même verrat, en estimant la fréquence des couples de truies sœurs ou demi-sœurs accouplées au même verrat. En France, obligation est faite à l'éleveur pour la mise à l'épreuve d'un verrat de choisir des truies qui ne soient ni sœurs ni demi-sœurs. On peut donc penser que le mode d'accouplement, dans la population, s'écarte peu des conditions de la panmixie.

De nombreuses estimations d'héritabilité pour les performances d'engraissement et de carcasse existent dans la littérature, depuis LUSH (I936). Nous nous sommes limités aux quatre caractères qui sont communs à toutes ces études et aux estimations obtenues dans les conditions d'une alimentation individuelle pour établir le tableau 7 . Si l'on met à part les dernières estimations, concernant le Landrace danois, où les héritabilités semblent avoir très nettement diminué ces dernières années, les valeurs trouvées pour le Large White français sont proches de celles des populations porcines étrangères. Par contre, il n'existe pas dans la littérature d'études génétiques concernant la composition corporelle, le coût 


\section{TABLEAU 7}

Résumé des estimations d'héritabilité

à partir de données d'épreuve de descendance en alimentation individuelle

\begin{tabular}{|c|c|c|c|c|c|}
\hline Auteurs & Race & $\begin{array}{c}\text { Gain } \\
\text { moyen }\end{array}$ & $\begin{array}{l}\text { Indice } \\
\text { de con- } \\
\text { somma- } \\
\text { tion }\end{array}$ & Longueur & $\begin{array}{l}\text { Epaisseur } \\
\text { de lard }\end{array}$ \\
\hline FREDEEN et JONSSON (I957) & Landrace danois & 0,50 & $0,5^{8}$ & 0,48 & 0,55 \\
\hline JoNSSON et KING (I962) .... & Landrace danois & 0,44 & 0,44 & 0,46 & 0,47 \\
\hline SMITH et coll. $(1962) \ldots \ldots$ & Large White anglais & $0,4 \mathrm{I}$ & 0,50 & 0,78 & 0,68 \\
\hline Jonsson $(1963) \ldots \ldots \ldots \ldots$ & Landrace danois & 0,22 & 0,32 & 0,37 & 0,45 \\
\hline Jonsson (I965) ......... & Landrace danois & 0,06 & 0,14 & 0,52 & 0,65 \\
\hline SMITH et Ross (1965) ... & Landrace anglais & $0,4 \mathrm{I}$ & 0,48 & 0,87 & 0,74 \\
\hline Présente étude $\ldots \ldots \ldots$ & Large White français & 0,54 & 0,48 & 0,72 & 0,65 \\
\hline
\end{tabular}

N.B. - Dans les cas où des estimations par sexe existaient, la moyenne des deux a été considérée.

de la dissection d'une carcasse de porc ne permettant pas, en général, de recueillir un nombre suffisant de données. La découpe commerciale standardisée est un palliatif. Il est cependant intéressant de connaître le poids des morceaux car ceux-ci sont directement liés à la valeur économique de la carcasse. Plutôt que de considérer l'ensemble des morceaux maigres et l'ensemble des morceaux gras, ce qui se fait généralement, la connaissance des paramètres relatifs à chacun des morceaux permet d'orienter d'une manière plus précise la sélection vers le type de carcasse souhaité. Il apparaît ainsi, au vu de nos résultats, qu'il est plus facile de développer par sélection la longe que le jambon, ce dernier morceau étant d'ailleurs constitué, pour une part non négligeable, de tissu gras.

Les corrélations génétiques entre la consommation et les critères de carcasse sont faibles, à l'exception du poids net. Par contre la durée d'engraissement est en corrélation plus forte avec les diverses mesures de carcasse et une sélection pour réduire cette durée doit tendre à produire des carcasses plus grasses. Il semble donc préférable de sélectionner sur l'indice de consommation. Que l'on sélectionne sur la durée ou sur la consommation, le rendement en carcasse doit diminuer. Notons quand même que toutes ces corrélations sont relativement faibles. Bien que le simple examen des corrélations génétiques ne permette pas de prévoir l'évolution résultant d'une sélection sur un indice combinant plusieurs caractères, il ne semble pas y avoir d'incomptabilité majeure entre l'amélioration de la vitesse de croissance et de l'efficacité alimentaire et la réduction de l'adiposité de la carcasse. Jonsson (I965), Smith et al. (I962), Smiтh et Ross (I965) aboutissent à des conclusions analogues.

L'analyse en composantes principales a été appliquée chez le Porc par Smith et al. (I962) et Smith et Ross (I965). Ces auteurs notent, comme dans cette étude, une similitude des composantes phénotypiques et génétiques et une première composante fortement associée aux mesures d'adiposité, mais leurs analyses ne 
font pas apparaître de composante liée à la fois à la croissance et à l'indice de consommation. I1 serait cependant imprudent de tirer des conclusions d'une telle comparaison, car on sait que les résultats de ce genre d'analyse dépendent, pour une large part, des variables prises en considération. La méthode a plutôt une valeur descriptive. Elle permet d'interpréter un ensemble de corrélations ainsi que de comparer entre elles plusieurs populations soumises aux mêmes mesures. Cependant, il faut signaler que 1'obtention de composantes génétiques n'est pas toujours possible, car la méthode des composantes principales suppose qu'aucune. des valeurs propres de la matrice ne soit négative, ce qui peut se produire dans le cas des corrélations génétiques. L'un des lecteurs m'a signalé une méthode proposée par Hashiguchi et MoRIshima (I969) qui permet de contourner cette difficulté. Dans cette étude, seule la dernière valeur propre de la matrice des corrélations génétiques chez les mâles est négative (et faible), et cette difficulté a donc pu être ignorée. Rouvier (I969) a, d'autre part, montré 1'intérêt des composantes génétiques pour calculer un indice de sélection dans le cas où l'importance économique relative des différents caractères est difficile à évaluer. Ainsi la première composante génétique fournit la combinaison linéaire de variance génétique maximum. Ici cette première composante principale exprime les variations de 1'adiposité en opposition avec celle des poids de certains morceaux (pieds et surtout longe). On peut donc raisonnablement penser que, dans la population considérée, on pourrait modifier de façon importante par sélection, la répartition des différents morceaux dans la carcasse et notamment augmenter le poids de la longe au détriment de la bardière.

$$
\text { Reçu pour publication en mai } 1970 .
$$

\section{REMERCIEMENTS}

L'auteur tient à remercier les $D^{\text {rs }}$ J. W. B. KING (A.B.R.O.-Edimbourg) et R. Rouvier (I.N.R.A.-Jouy-en-Josas) de leurs remarques et suggestions à la lecture de ce texte.

\section{SUMMARY}

PROGENY-TEST IN FRENCH “ LARGE WHITE” PIG FROM I953 TO I966

\section{I. - ANALYSIS OF VARIATION}

From a sample of 2657 females and 1515 castrated males, tested in the Jouy-en-Josas progenytesting station, heritabilities and genetic correlations in the French Large White breed have been estimated for food eaten and time on test between 25 and $100 \mathrm{~kg}$ liveweight, for weight of different cuts, length and backfat thickness on the carcass at $100 \mathrm{~kg}$ liveweight. These estimates are very similar to those obtained for the same breed in Great Britain. A principal component analysis has been carried out on genetic and phenotypic correlation matrices, for each sex separately. The results are very similar in the four cases. The first two components account for about 50 p. 100 of the total variance. The first is associated with measurements of fatness and the second with food eaten and time on test. 


\section{RÉFÉRENCES BIBLIOGRAPHIQUES}

Czajewska S. J., Ollivier L., r968. Note sur l'utilisation des résultats des épreuves de descendance par les sélectionneurs de porcs en France de 1956 à 1965. Ann. Génét. Sél. anim., 1, 71-77.

Dickerson G. E., Hazel L. N., I944. Effectiveness of selection on progeny performance as a supplement to earlier culling in livestock. J. agric. Res., 69, 459-476.

Falconer D. S., 1960. Introduction to Quantitative Genetics. Oliver and Boyd. London.

FAlConer D. S., 1963. In Burdette W. J., Methodology in Mammalian Genetics, 193-216. Holden Day. San Francisco.

FLock D., I967. Berechnung des durschnittlichen Verwandtschaftsgrades aus der Häufigkeit von Geschwisterpaaren. Z. Tierz. und ZüchtBiol., 83, 235-239.

Fredeen H. T., Jonsson P., I967. Genetic variance and covariance in Danish Landrace swine as evaluated under a system of individual feeding of progeny test groups. Z. Tierz. ZüchtBiol., 70, 348-363.

GILlors M., 1964. La relation d'identité en génétique. Thèse Fac. Sci., Paris.

HARRIS D. L., I964. Genotypic covariances between inbred relatives. Genetics, 50, I3I9-1348.

HASHiguchi S., Morishima H., 1969. Estimation of genetic contribution of principal components to individual variates concerned. Biometrics, 25, 9-I5.

Hotelling H., 1933. Analysis of a complex of statistical variables into principal components. J. Educ. Psych., 24, 4I7-44I, 498-520.

Jonsson P., r963. Danish pig progeny testing results. Z. Tierz. ZüchtBiol., 78, 205-252.

Jonsson P., 1965. Analyse af egenskaber hos svin af Dansk Landrace med en historisk indledning. 350. beretning fra forsöglaboratoriet, $490 \mathrm{pp}$.

Jonsson P., KING J. W. B., 1962. Sources of variation in Danish Landrace pigs at progeny-testing stations. Acta. A gric. scand., 12, 68-80.

Kempthorne O., 1956. An Introduction to Genetic Statistics. John Wiley and Sons. New-York.

I_ANGHOLz H. J., 1965. Das züchterische Hilfsmittel der stationären Nachkommenprïfung beim Schwein. I Systematische Einflüsse auf die Ergebnisse aus der Mastleistungsprüfung für Schweine. Acta. A gric. scand., 15, Ir5-I44.

LANGHOLz H. J., 1965. Das züchterische Hilfsmittel der stationären Nachkommenprüfung beim Schwein. II Die Verwendung eines gleitenden Stationsmittels zur unmittelbaren Erfassung temporärer Umwelt veranderungen und die genetische Aussage der in der stationären Nachkommenprüfung beobachteten L,eistungsverschiebung. Acta Agric. Scand., 15, 181-203.

I.ANGHOLz H. J., 1966. Das züchterisahe Hilfsmittel der stationären Nachkommenprüfung beim Schwein. III. Heritabilitäten und genetische Korrelationen beim norwegischen Landschwein. Acta Agric. Scand., 16, 97-114.

LAUPREChT E., WALTER E., I96o. Uber einige Umwelteinflüsse auf die Mast-und Schlachteigenschaften des Schweines bei dänischen Mastprüfungsgruppen, Archiv für Tierz., 3, 3-25.

I,UsH J. L., 1936. Genetic aspects of the Danish system of progeny testing swine. Iow Agr. Exp. Sta. Res. Bull. (204).

Ollivier L., 1969. Paramètres génétiques du porc Large White français. Journées de la Recherche porcine. Paris 20-2r février, 7-12.

Rouvier R., I969. Pondération des valeurs génotypiques dans la sólection par index sur plusieurs caractères. Biometrics, 25, 295-307.

Rovvier R., Ricard F. H., I965. Étude des mesures de conformation du poulet. II. Recherche des composantes de la variabilité morphologique du poulet vivant. Ann. Zootech., 14, 213-227.

Smitr C., 1965. Results of pig progeny testing in Great Britain. Anim. Prod., 7, I33-140.

Sмith C., Ross G. J. S., I965. Gentic parameters of British Landrace bacon pigs. Anim. Prod., 7, 29I-30r.

Smith C., KING J. W. B., Gilbert N., rg62. Genatic parameters of British Large White bacon pigs. Anim. Prod., 4, 128-143.

TAYlor St. C. S., Rollivs W. C., 1963. Body size and conformation in identical twin cattle. Anim. Prod., 5, 77-86.

Vissac B., Poutous M., Tassencourt L., I962. Analyse et décomposition des variances et covariances dans le cas d'un schéma hiérarchique. Programme 62021 de la Station centrale de Génétique animale. 